Check for updates

Cite this: RSC Adv., 2017, 7, 34366

Received 14th April 2017

Accepted 19th June 2017

DOI: $10.1039 / \mathrm{c} 7 \mathrm{ra04250g}$

rsc.li/rsc-advances

\section{Synergetic effect of CuSaZnS nanostructures on photocatalytic degradation of organic pollutant under visible light irradiation $\dagger$}

\author{
S. Harish, ${ }^{a}$ J. Archana, ${ }^{\star b}$ M. Navaneethan, (D) *ab S. Ponnusamy, ${ }^{* b}$ Ajay Singh, ${ }^{c}$

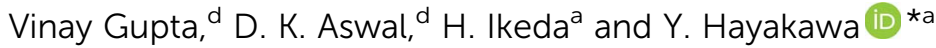

\begin{abstract}
Ultrafast visible light active CuS/ZnS nanostructured photocatalysts were synthesized by a hydrothermal method. The effect of the CuS concentration on the morphological, structural and optical properties of ZnS nanostructures were investigated. X-ray diffraction analysis indicated the formation of CuS/ZnS phases with good crystallinity. The presence of ZnS on CuS was confirmed by X-ray photoelectron spectroscopy, elemental mapping, scanning electron microscopy and high resolution transmission electron microscopy analyses. CuS/ZnS nanocomposites efficiently decomposed methylene blue (MB) upon irradiation with visible light. The degradation time was 3 times faster than that of bare ZnS and CuS. The scavenger analysis results revealed that photogenerated superoxide $\left(\mathrm{O}_{2}^{-\cdot}\right)$ radicals were the main reactive species for the degradation of MB. The maximum degradation efficiency of $95.51 \%$ was observed within $5 \mathrm{~min}$ for samples of $0.050 \mathrm{M} \mathrm{CuS/ZnS} \mathrm{composites.} \mathrm{The} \mathrm{obtained} \mathrm{results} \mathrm{suggested} \mathrm{that}$ the enhanced photocatalytic activity was due to the formation of CuS/ZnS heterojunctions which led to the efficient separation of the photoinduced carriers.
\end{abstract}

\section{Introduction}

Photocatalysts have been explored for their utility in environmental applications such as pollutant dissociation, electric energy conversion devices, and water-splitting applications which are required for the quality of our life. ${ }^{1-6}$ Photocatalysis provides a potential strategy to decompose contaminants by light-driven chemical processes over the surface of the photocatalyst. Honda and Fujishima's pioneering work of photocatalytic water splitting on $\mathrm{TiO}_{2}$ electrodes has attracted widespread attention in clean, environmentally friendly and economical processes. ${ }^{7,8}$ Various semiconductor photocatalysts, including metal oxides, metal hydroxides, oxynitrides, sulfides and metal-free semiconductors, have been investigated for water purification..$^{9-12}$ Among the photocatalysts, zinc sulfide $(\mathrm{ZnS})$ has been studied extensively by several researchers. ${ }^{13} \mathrm{ZnS}$ is an important II-VI group

${ }^{a}$ Research Institute of Electronics, Shizuoka University, 3-5-1 Johoku, Naka-ku, Hamamatsu, Shizuoka 432-8011, Japan. E-mail: mpnavaneethan@yahoo.co.in; royhaya@ipc.shizuoka.ac.jp; Fax: +81 53 4781338; Tel: +81 534781338

${ }^{b}$ Center for Materials Science and Nano Devices, Department of Physics and Nanotechnology, SRM University, Kattankulathur, Chennai, 603203, Tamil Nadu, India. E-mail: jayaram.archana@gmail.com; suruponnus@gmail.com; Fax: +91 44 27456255; Tel: +914427452818

${ }^{c}$ Technical Physics Division, Bhabha Atomic Research Center, Mumbai 400 085, India ${ }^{d}$ Organic and Hybrid Solar Cell Group, CSIR-National Physical Laboratory, New Delhi, India

$\dagger$ Electronic supplementary information (ESI) available. See DOI: $10.1039 / \mathrm{c} 7 \mathrm{ra04250g}$ semiconductor, which can rapidly produce electron-hole pairs by photoexcitation and thus create high negative potentials of the excited electron. The theoretical efficiency of the photo-carrier generation of $\mathrm{ZnS}$ is much higher than that of $\mathrm{TiO}_{2}$ because of a direct band gap. ${ }^{14}$ However, the efficiency of $\mathrm{ZnS}$ is impeded by a wide band gap energy $\left(E_{\mathrm{g}}=3.66 \mathrm{eV}\right)$ that strictly precludes the utilization of visible light and results in a reduced viability of ZnS as a visible light photocatalyst. These photocatalysts are mostly active only under UV light, which only occupies $4 \%$ of the total sunlight, and thus restricts its practical application. ${ }^{15}$

A composite with two semiconductors having a $\mathrm{p}-\mathrm{n}$ junction can induce the transition of the optical responsibility from UV to the visible region and separate the electron-hole pairs. ${ }^{\mathbf{1 6 , 1 7}}$ Thus, an improved charge separation, increased charge carrier lifetime and efficient interfacial charge transfer from the catalyst to the adsorbed substance are achieved in the presence of composite semiconductors as the photocatalyst, resulting in an enhanced photocatalytic activity. It is known that the photocatalytic activity of a photocatalyst mainly depends on an amount of the photoexcited active species and the separation efficiency of the photoexcited electron-hole pairs. ${ }^{18}$ The formation of the $\mathrm{p}-\mathrm{n}$ junction in a photocatalytic system provides an internal electrostatic field with a direction from the n-type semiconductor to the p-type semiconductor. The photoexcited electron-hole pairs would be thus effectively separated by moving the electron and hole in opposite directions and their recombination can be minimized. ${ }^{19-21}$ Therefore, it is desirable to design an efficient visible 
light driven photocatalyst in which one component is a p-type semiconductor and the other is an n-type semiconductor.

Composite nanostructures have been studied for these photocatalytic applications. For example, Lee et al. fabricated CuS (ptype) and $\mathrm{ZnO}$ (n-type) nanoarrays on a stainless steel mesh and applied them as a visible light photocatalyst for the decomposition of acid orange $7 .{ }^{22}$ Zhang et al. showed that p-type NiO and n-type $\mathrm{ZnO}$ heterojunction nanofibres exhibited a superior photocatalytic activity over pure $\mathrm{NiO}$ and $\mathrm{ZnO}$ nanofibres for the degradation of Rhodamine B. The separation of photogenerated electron-hole pairs was highly enhanced. ${ }^{23}$ Soltani et al. synthesized ZnS and CdS nanoparticles which extended the light absorption in the visible region and showed an increased stability against photo-corrosion. ${ }^{24} \mathrm{Babu}$ et al. synthesized $\mathrm{Cu} /$ $\mathrm{TiO}_{2}$ core-shell nanowires with $3 \% \mathrm{Cu} / \mathrm{TiO}_{2}$ and indicated the highest degradation rate of methyl orange. ${ }^{25}$ Sunita et al. synthesized $\mathrm{TiO}_{2} / \mathrm{CuS}$ core-shell nanostructures. Methylene blue was degraded within $60 \mathrm{~min}$ of irradiation. ${ }^{26}$ Basu et al. prepared $\mathrm{ZnO} / \mathrm{CuS}$ heterostructure by decorating $\mathrm{CuS}$ nanostructures on the surface of $\mathrm{ZnO}$ nanotubes by a wet chemical method. They showed that the $\mathrm{ZnO} / \mathrm{CuS}$ heterostructure efficiently decomposed methylene blue (MB) upon irradiation of visible light at room temperature. ${ }^{27}$ The composites facilitated charge transfer across heterojunctions, effectively improving the separation of photogenerated electron-hole pairs. ${ }^{28}$ The above research clearly demonstrated that the coupling of semiconductors significantly enhances their photocatalytic activities. Copper sulfide (CuS) is a p-type semiconductor with a narrow band gap $\left(E_{\mathrm{g}}=2.2 \mathrm{eV}\right)$ which could be a highly efficient and stable visible-light photocatalyst for pollutant treatment. ${ }^{29-31}$ However, the rapid recombination of the photogenerated electron-hole pairs of CuS restricts the improvement of its degradation efficiency as a photocatalyst. The formation of a heterojunction between $\mathrm{ZnS}$ and $\mathrm{CuS}$ in the nanocomposite photocatalyst is expected to promote the transfer of photogenerated electrons and holes between $\mathrm{ZnS}$ and CuS, which suppresses their recombination and enhances the photocatalytic activity. ${ }^{32-35}$ Therefore, CuS/ZnS nanocomposites can be a promising material as a photocatalyst because of their physical and chemical properties.

In the present work, a novel approach is reported for the formation of well-connected $\mathrm{ZnS} / \mathrm{CuS}$ nanostructures by

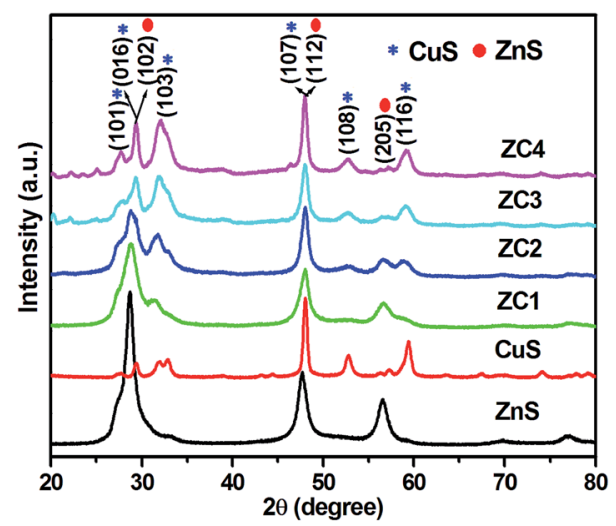

Fig. 1 XRD patterns of ZnS, CuS and CuS/ZnS nanocomposites. a hydrothermal method. The effect of CuS concentration on the phase and morphology of the composites is investigated. The functional properties of the $\mathrm{ZnS} / \mathrm{CuS}$ nanocomposites are studied by X-ray diffraction (XRD), field-emission scanning electron microscopy (FESEM), transmission electron microscopy (TEM) and X-ray photoelectron spectroscopy (XPS). The photocatalytic activity of the synthesized materials is obtained by quantifying the rate of $\mathrm{MB}$ degradation in an aqueous suspension under visible light irradiation. The kinetics and photocatalytic mechanism of $\mathrm{ZnS} / \mathrm{CuS}$ nanocomposites are proposed.

\section{Experimental procedure}

All the chemicals were purchased from Wako chemicals (Japan) and used without further purification. In a typical synthesis,


Fig. 2 XPS spectra of (a) Zn 2p, (b) Cu 2p and (c) S 2s states. 
$0.1 \mathrm{~mol} \mathrm{~L}^{-1}$ of copper acetate was dissolved in $100 \mathrm{~mL}$ of deionized water and allowed to stir until complete dissolution of the compound. $1 \mathrm{~mL}$ of triethylamine (TEA) was added to the above solution as a stabilizing agent. $0.1 \mathrm{~mol} \mathrm{~L}^{-1}$ of thioacetamide was added and allowed to stir for $12 \mathrm{~h}$. Finally, the mixture was transferred to a Teflon-lined autoclave and maintained at $150{ }^{\circ} \mathrm{C}$ for $12 \mathrm{~h}$ in a hot air oven. The obtained precipitate was separated by centrifugation, and washed with deionized water and ethanol several times. The samples were termed CuS. For the synthesis of ZnS nanostructures, the same procedure was followed with a small modification. $0.1 \mathrm{~mol} \mathrm{~L}^{-1}$ of zinc acetate was added instead of copper acetate and the sample was termed $\mathrm{ZnS}$.

\subsection{Synthesis of $\mathrm{CuS} / \mathrm{ZnS}$ nanocomposites}

$0.1 \mathrm{~mol} \mathrm{~L}^{-1}$ of zinc acetate was dissolved in $100 \mathrm{~mL}$ of deionized water and allowed to stir until complete dissolution of the compound. Copper acetate of different mol percentages $(0.025,0.050,0.075$ and $0.1 \mathrm{M})$ was added to the zinc acetate solution under continuous stirring. $1 \mathrm{~mL}$ of TEA and $0.1 \mathrm{~mol}$ $\mathrm{L}^{-1}$ of thioacetamide were added and allowed to stir for $12 \mathrm{~h}$. Finally, the mixture was transferred to a Teflon-lined autoclave and maintained at $150{ }^{\circ} \mathrm{C}$ for $12 \mathrm{~h}$ in a hot air oven. The obtained product was separated by centrifugation, and washed with deionized water and ethanol several times. The obtained product was dried at $80{ }^{\circ} \mathrm{C}$ for $1 \mathrm{~h}$. The samples were termed as ZC1, ZC2, ZC3 and ZC4 for the copper acetate concentrations of $0.025,0.050,0.075$ and $0.1 \mathrm{M}$, respectively.

\subsection{Characterization techniques}

The obtained products were characterized by XRD using a Rigaku X-ray diffractometer (RINT 2200, Japan) with CuKa radiation and $0.02^{\circ} \mathrm{s}^{-1}$ step interval. The morphology and particle size of the products were assessed by FESEM (JEOL JSM $7001 \mathrm{~F}$ microscope) at an accelerating voltage of $15 \mathrm{kV}$ and TEM (JEOL JEM 2100F microscope) at an accelerating voltage of 200 $\mathrm{kV}$. UV-visible absorption spectra were performed by a Shimadzu 3101 PC spectrophotometer (Japan). XPS spectra were measured by a Shimadzu ESCA 3400 .

\subsection{Photocatalytic studies}

Evaluation of the photocatalytic performance of CuS/ZnS composites was explored by examining the photo-assisted degradation of $\mathrm{MB}$ under visible light illumination [xenon light source (MAX-303, Asahi Spectra)]. The photocatalytic ability tests were accomplished in a $50 \mathrm{~mL}$ glass beaker. In the photocatalytic reaction, the dye concentration was fixed at $10 \mathrm{ppm}$, and the photocatalyst of a known dosage $\left(75 \mathrm{mg} \mathrm{L}^{-1}\right)$ was added to the above dye solution. The suspensions were stirred to achieve an absorption-desorption equilibrium state for the solution which was required to be kept in the dark before light irradiation. ${ }^{36,37}$ The reaction mixture was irradiated with stirring under the xenon lamp positioned at $21 \mathrm{~cm}$ above the reaction mixture. A blank experiment was executed either in the absence of sample (photocatalyst) or in the dark to determine that degradation of $\mathrm{MB}$ is truly operated by a photocatalytic mechanism. At regular time intervals, $3 \mathrm{~mL}$ of the suspension was collected, centrifuged, and analysed by a UV-vis spectrometer. The MB degradation was estimated from the decrease in

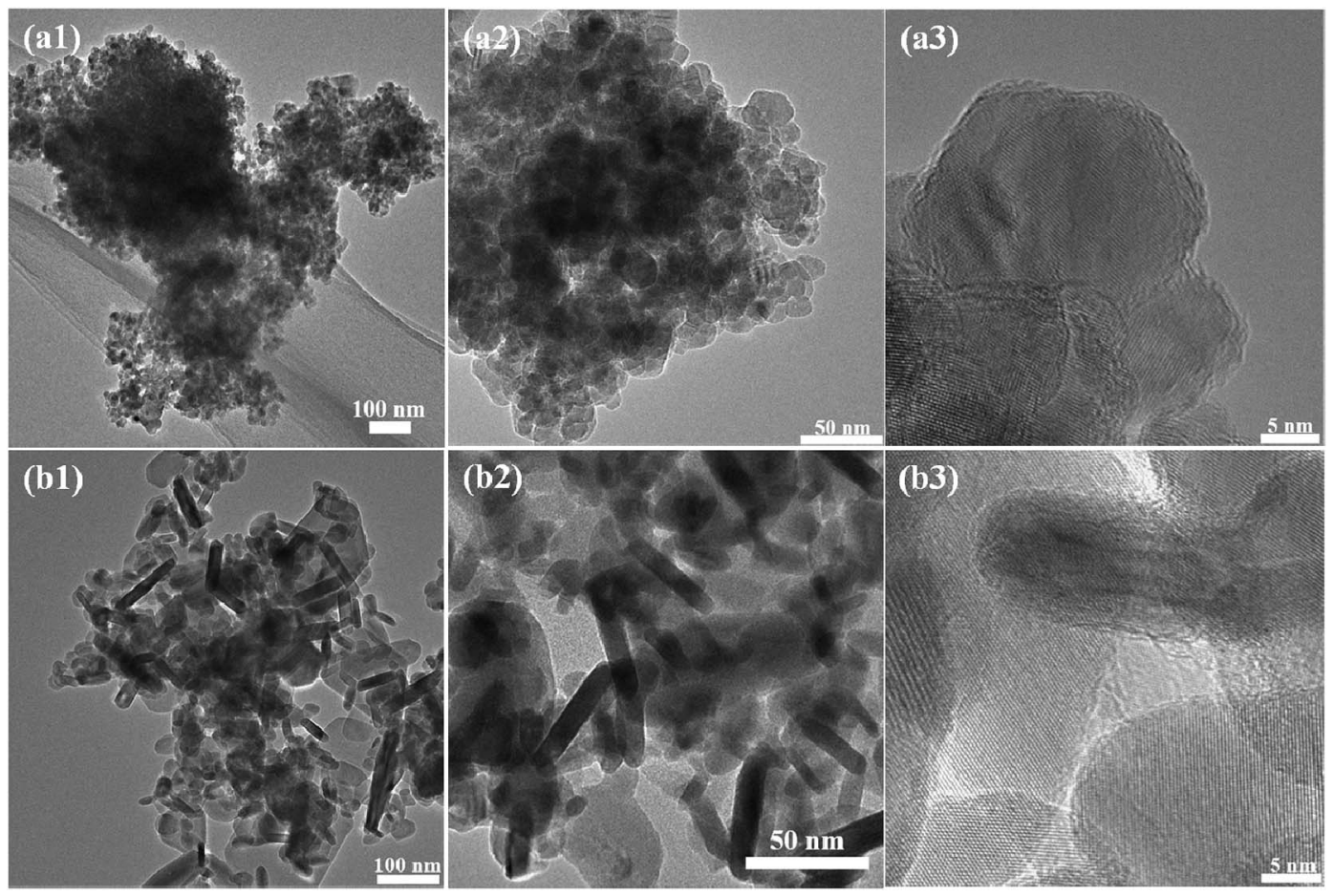

Fig. 3 TEM and HRTEM images of ZnS (a1-a3) and CuS (b1-b3). 
the intensity of the associated characteristic band absorption at $664 \mathrm{~nm}$. The photodegradation percentage of MB was calculated using the following equation: ${ }^{38}$

$$
D(\%)=\frac{C_{0}-C_{t}}{C_{0}} \times 100,
$$

where $C_{0}$ refers to the absorption of $\mathrm{MB}$ after the absorption equilibrium is achieved prior to the visible light illumination and $C_{t}$ is the concentration of $\mathrm{MB}$ at time interval $t$ under visible light illumination.

To achieve a full understanding of the photocatalytic mechanism of $\mathrm{MB}$ over the $\mathrm{CuS} / \mathrm{ZnS}$ composites, trapping experiments utilizing active species trappers were conducted. Different scavengers were used to trap photogenerated electrons and holes in the photocatalysis. Potassium persulfate $\left(\mathrm{K}_{2} \mathrm{~S}_{2} \mathrm{O}_{8}\right)$ and benzoic acid (BA) were used to trap

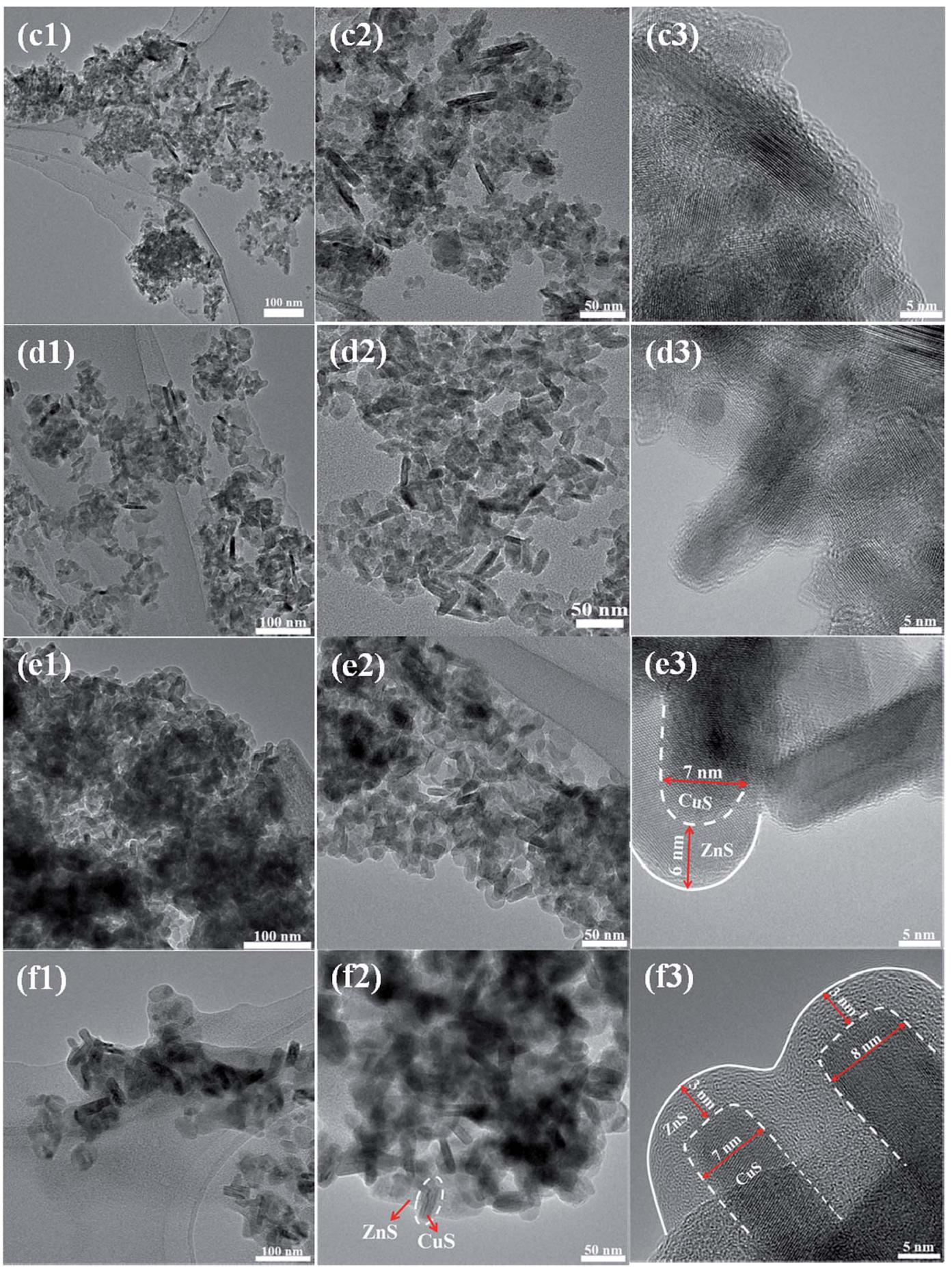

Fig. 4 TEM and HRTEM images of CuS/ZnS composites ZC1 (c1-c3), ZC2 (d1-d3), ZC3 (e1-e3) and ZC4 (f1-f3). 
electrons and holes, keeping all the other conditions unaltered.

\section{Results and discussion}

XRD patterns of pure $\mathrm{ZnS}$, CuS and $\mathrm{CuS} / \mathrm{ZnS}$ composites are shown in Fig. 1. The XRD pattern of pure ZnS exhibited several diffraction peaks at $28.70^{\circ}, 47.61^{\circ}$ and $56.55^{\circ}$, which corresponded to the (102), (112) and (205) planes of the cubic phase of ZnS (JCPDS card no. 89-2194). CuS sample possessed diffraction peaks at $27.51^{\circ}, 29.37^{\circ}, 31.74^{\circ}, 48.11^{\circ}, 52.67^{\circ}$ and $59.42^{\circ}$ which corresponded to (101), (016), (103), (107), (108) and (116) planes of the hexagonal phase of CuS (JCPDS card no. 24-0060). The samples of ZC1-ZC4 showed the XRD pattern of CuS/ZnS composites. The sample ZC1 showed mixed planes that belonged to the (103) and (116) planes of CuS, whereas the samples with a higher CuS concentration, i.e. ZC2 to $\mathrm{ZC} 4$, possessed all the peaks of the cubic $\mathrm{ZnS}$ and hexagonal CuS patterns. This depicted the formation of $\mathrm{ZnS} / \mathrm{CuS}$ composites. The intensity of the $\mathrm{ZnS}$ peaks decreased with an increase in the concentration of CuS. ${ }^{39,40}$ All the diffraction peaks indicated that the materials crystallized well as composites. The structures of $\mathrm{ZnS}$ and CuS in all the samples were identified as cubic (JCPDS card no. 89-2194) and hexagonal (JCPDS card no. 89-2194). ${ }^{41}$

The elemental composition and chemical states of ZnS, CuS and $\mathrm{CuS} / \mathrm{ZnS}$ composite samples were analysed using XPS. The high-resolution XPS spectra of $\mathrm{Zn} 2 \mathrm{p}, \mathrm{Cu} 2 \mathrm{p}$ and $\mathrm{S} 2 \mathrm{~s}$ are shown in Fig. 2(a), (b) and (c), respectively. The core-level spectra of the $\mathrm{Zn} \mathrm{2p}$ state of pure $\mathrm{ZnS}$ displayed two peaks centred at $1022.97 \mathrm{eV}$ and $1045.92 \mathrm{eV}$ which corresponded to $\mathrm{Zn} 2 \mathrm{p}_{3 / 2}$ and $\mathrm{Zn} 2 \mathrm{p}_{1 / 2}$ suggesting the existence of $\mathrm{Zn}^{2+} .^{42,43}$ The energy difference between the two binding energies was $22.95 \mathrm{eV}$, which was in good agreement with the standard value. ${ }^{\mathbf{4 4 4 5}}$ The peak positions of samples ZC1 were shifted from 1022.97 to $1022.87 \mathrm{eV}$ and from 1045.92 to $1045.82 \mathrm{eV}$ when compared to pure ZnS. When the CuS concentration increased, the peak positions were shifted to $1021.69 \mathrm{eV}$ and $1044.75 \mathrm{eV}$ for sample ZC2, $1021.69 \mathrm{eV}$ and $1044.64 \mathrm{eV}$ for sample ZC3 and $1021.48 \mathrm{eV}$ and $1044.53 \mathrm{eV}$ for sample ZC4. A slight shift in the binding energies of the $\mathrm{CuS} / \mathrm{ZnS}$ composite samples was observed due to the electronic exchange caused by the difference the electronegativity of zinc (1.6) and copper (1.9) elements. ${ }^{46}$

As shown in Fig. 2(b), two typical peaks at $933.52 \mathrm{eV}$ and $953.44 \mathrm{eV}$ corresponded to $\mathrm{Cu} 2 \mathrm{p}_{3 / 2}$ and $\mathrm{Cu} 2 \mathrm{p}_{1 / 2}$, respectively. These peaks have the same binding energies as the $\mathrm{Cu} 2 \mathrm{p}$ orbital of the $\mathrm{Cu}^{2+}$ state. ${ }^{47,48}$ The peak positions of sample ZC1 were shifted from $933.52 \mathrm{eV}$ to $933.34 \mathrm{eV}$ and from $953.44 \mathrm{eV}$ to 953.26 eV when compared to pure CuS. Similar peak shifts were observed in the binding energies of CuS/ZnS composites from 933.34 to $932.26 \mathrm{eV}$ and from 953.26 to $952.10 \mathrm{eV}$, respectively. The binding energy of the S 2 s spectra centred at $224.69 \mathrm{eV}$ is the characteristic value for metal sulfides (Fig. 2(c)). ${ }^{49}$ A similar peak shift from $224.69 \mathrm{eV}$ to $223.98 \mathrm{eV}$ was observed in the composite samples. By the above analyses, it can be concluded that there is a strong interaction between $\mathrm{ZnS}$ and $\mathrm{CuS}$ in the composites.

The morphology of the sample was observed by FESEM and TEM analysis, as shown in Fig. 3 and 4. Fig. 3(a1)-(a3) shows the FESEM and TEM images of pure ZnS. The pure ZnS possessed spherical nanoparticles. All the particles were in the range of 10-20 $\mathrm{nm}$ in diameter with crystalline fringes, as observed by HRTEM in Fig. 3(a3). Whereas the pure CuS (Fig. 3(b1)-(b3)) exhibited a morphology of nanorods. The size of the nanorods was in the range of about 15-20 nm in width and 100-300 $\mathrm{nm}$ in

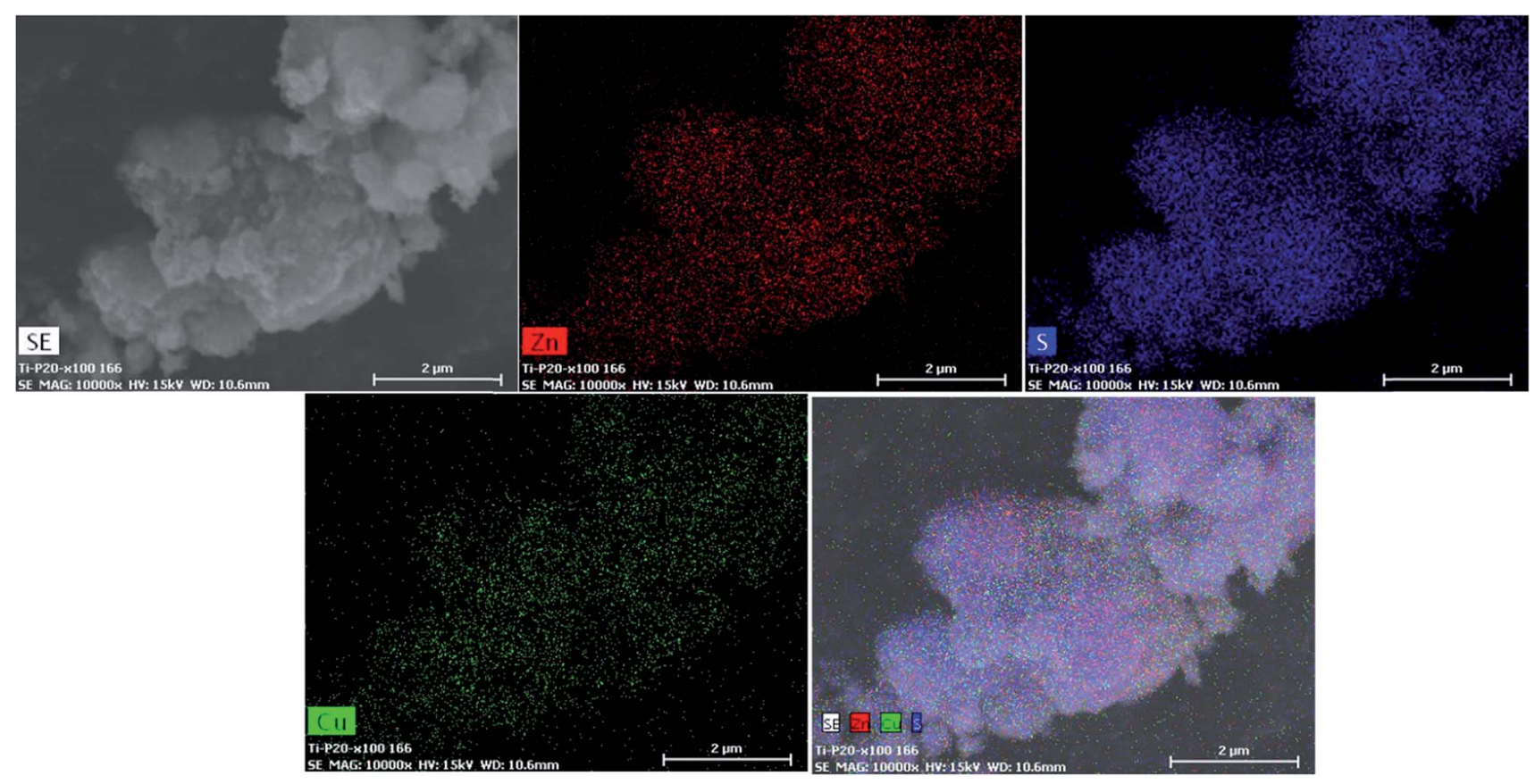

Fig. 5 EDS elemental mapping of the ZC2 sample. 

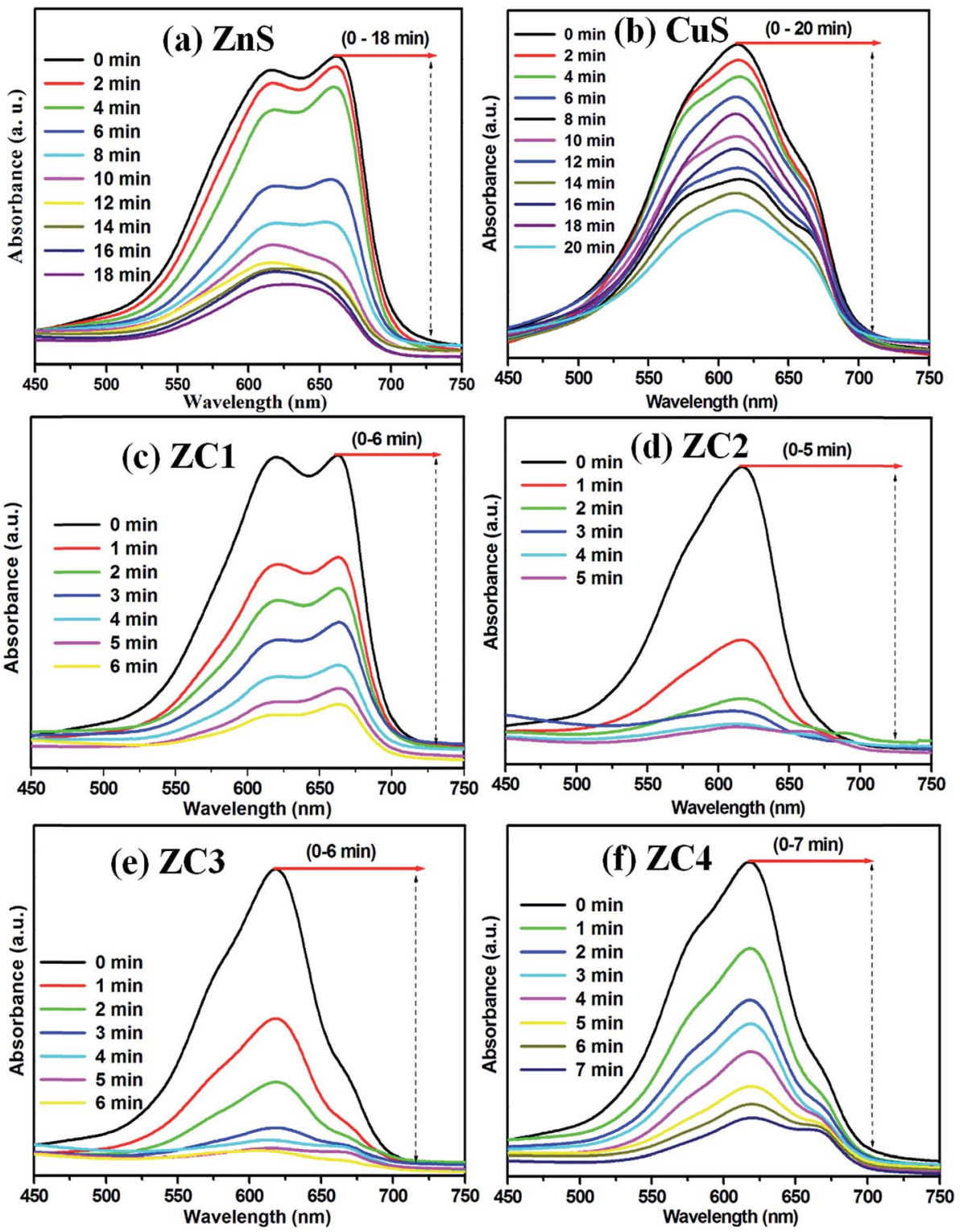

Fig. 6 UV absorption spectra of MB (a) ZnS, (b) CuS, (c) ZC1, (d) ZC2, (e) ZC3 and (f) ZC4.

length. When the CuS concentration was $0.025 \mathrm{M}$, the $\mathrm{ZnS}$ nanoparticles were partially coated on the surface of the CuS nanorods, as shown in Fig. 4(c1)-(c3). As the CuS concentration was increased to 0.050 ( $\mathrm{ZC} 2)$, the surface of the nanorods was fully covered by ZnS nanoparticles, as shown in Fig. 4(d1)-(d3). With further increases in the concentration of CuS to 0.075 (ZC3) and 0.1 M (ZC4), the CuS nanorods were embedded in the $\mathrm{ZnS}$ nanostructures, as shown in Fig. 4(e1)-(f3). The highresolution TEM (HRTEM) images obtained from the edge of individual nanorods revealed the formation of heterojunctions of $\mathrm{ZnS}$ and $\mathrm{CuS}$ nanoparticles. Fig. 5 shows an element mapping analysis of $\mathrm{Zn}, \mathrm{Cu}$ and $\mathrm{S}$ for the $\mathrm{ZC} 2$ sample. The signals for $\mathrm{Zn}$, $\mathrm{Cu}$ and $\mathrm{S}$ were uniform, indicating the homogeneous distribution of $\mathrm{Zn}, \mathrm{S}$ and $\mathrm{Cu}$ elements in the $\mathrm{CuS} / \mathrm{ZnS}$ composites.

MB photodegradation is of great significance for environmental pollutant treatment, and it is commonly used as a model pollutant for evaluating the degradation efficiency of a photocatalyst. The time-dependent UV-Vis absorption spectrum of $\mathrm{CuS} / \mathrm{ZnS}$ composites under visible light irradiation is shown in Fig. 6(a)-(f). Fig. 6(a) and (b) show the time-dependent UV absorption spectra of bare $\mathrm{ZnS}$ and CuS, respectively. MB was completely decomposed in 20 and $18 \mathrm{~min}$, respectively. Fig. 6(c)-(f) show the photodegradation of samples ZC1-ZC4, respectively. The initial absorbance of the peak disappeared completely after 5 min of irradiation for sample ZC2, as shown in Fig. 6(d). As the lower concentration of the CuS is $0.025 \mathrm{M}$, the photodegradation time increased to $6 \mathrm{~min}$, as shown in Fig. 6(c), and when the concentration increased to 0.075 and $0.1 \mathrm{M}$, the degradation time increased to 6 and $7 \mathrm{~min}$, as shown in Fig. 6(e) and (f), respectively. It can be clearly seen that the photocatalytic activities of the CuS/ZnS composites depended on the amount of CuS concentration. The highest photocatalytic 

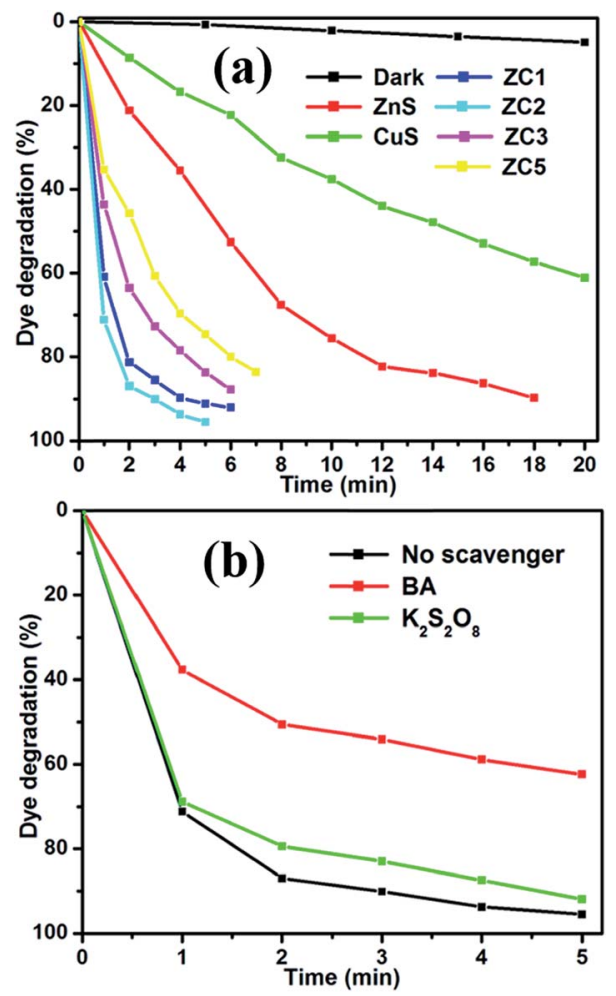

Fig. 7 Effect of dye degradation efficiency (a) time (min) vs. dye degradation (\%), and (b) MB over CuS/ZnS in the presence of various scavengers under visible light irradiation.

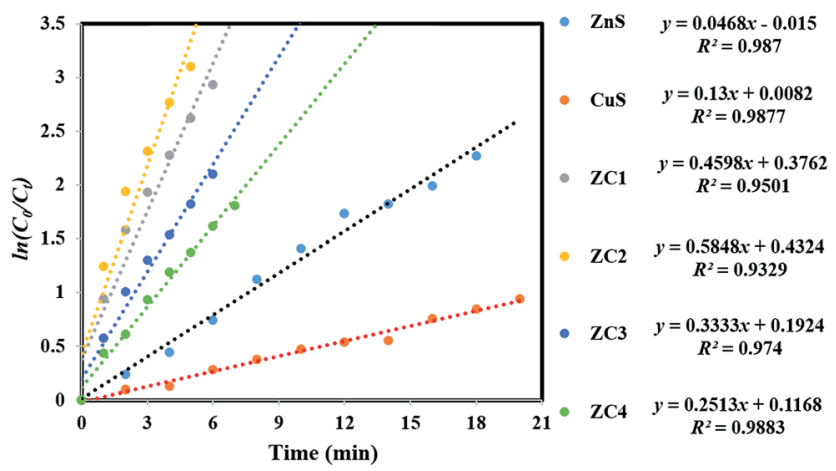

Fig. 8 Plots of $\ln \left(C_{0} / C_{t}\right)$ as a function of time (min) for the photodegradation of MB over the CuS/ZnS nanocomposites.

activity was obtained for the $0.025 \mathrm{M}$ sample (ZC2). Fig. 7(a) shows the blank experiment, carried out in the absence of a catalyst, with no change in the degradation of MB. This observation clearly shows that the degradation of MB occurred only with the photocatalyst present. The degradation percentages of MB in the presence of ZnS, CuS, ZC1, ZC2, ZC3 and ZC4 were $89.70,61.05,92.07,95.51,87.79$ and $83.65 \%$, respectively. The photocatalytic activity strongly depends on the initial concentration of $\mathrm{Cu}^{2+}$ in the ion-exchange reaction. The photocatalytic reaction of the pure sample is decreased and it is drastically increased after the incorporation of CuS in the composition. The addition of CuS formed a heterojunction,
Table 1 Observed pseudo-first order rate constants, $R^{2}$ values, maximum degradation (\%) and time required for maximum degradation of CuS/ZnS nanostructures

\begin{tabular}{lllll}
\hline Sample & $\begin{array}{l}K_{\text {app }} \\
(\text { CuS/ZnS })\end{array}$ & $R^{2}$ & $\begin{array}{l}\text { Maximum } \\
\text { degradation } \\
(\%)\end{array}$ & $\begin{array}{l}\text { Time taken for } \\
\text { maximum } \\
\text { degradation }(\mathrm{min})\end{array}$ \\
\hline ZnS & 0.0046 & 0.9870 & 89.70 & 20 \\
CuS & 0.1300 & 0.9877 & 61.05 & 18 \\
ZC1 & 0.4598 & 0.9501 & 92.07 & 06 \\
ZC2 & 0.5848 & 0.9329 & 95.51 & 05 \\
ZC3 & 0.3333 & 0.9740 & 87.79 & 06 \\
ZC4 & 0.2513 & 0.9883 & 83.65 & 07
\end{tabular}

resulting in a significant increase in the photocatalytic activity. Sample ZC2 shows the highest photocatalytic activity, and a further increase in the concentration of CuS leads to a decrease in the activity. The increased concentration of $\mathrm{CuS}$ may lead to a decrease in the interface and act as recombination centres of electron-hole pairs. Thus, the separation efficiency of the electron-hole pairs decreases with a decrease in the activity. Many results reported the same phenomena, such as $\mathrm{ZnS} / \mathrm{CuS}$ nanotubes, $\mathrm{CuS} / \mathrm{ZnS}$ nanowires, $\mathrm{Cu}-\mathrm{ZnS}, \mathrm{TiO}_{2} / \mathrm{ZuS}$ and $\mathrm{ZnO} /$ SnO..$^{50-54}$

Different reactive species, such as the hydroxyl radical ( $\left.{ }^{\circ} \mathrm{OH}\right)$ and superoxide radical ion $\left(\mathrm{O}_{2}^{-}\right)$, are generated in the photocatalysis process. The main reactive species are electrons and holes. To understand the photocatalysis process in-depth, different scavenging environments were studied. From the literature, it is apparent that benzoic acid and potassium persulfate can act as good electron and hole scavengers. ${ }^{55,56}$ Benzoic acid (BA) is used to trap electrons of the conduction band and potassium persulfate $\left(\mathrm{K}_{2} \mathrm{~S}_{2} \mathrm{O}_{8}\right)$ is used to trap holes of the valence band before the inclusion of the $\mathrm{CuS} / \mathrm{ZnS}$ photocatalyst. Fig. 7(b) shows the degradation of methylene blue in the presence of the hole scavenger, $\mathrm{K}_{2} \mathrm{~S}_{2} \mathrm{O}_{8}$. In the presence of the $\mathrm{O}_{2}{ }^{-}$ scavenger, only $5 \%$ was decreased compared with the scavenger free photocatalyst for MB. To examine the reactive species, another experiment was performed using $\mathrm{BA}$ as the ${ }^{\circ} \mathrm{OH}$ scavenger. As a result, only a $62 \% \mathrm{MB}$ degradation was observed instead of $95 \%$ for the scavenger free photocatalyst. This indicates that the superoxide radical was the most dominant species. Due to the scavenging of the superoxide radical, the probability of electron-hole pair recombination diminishes and the rate of reaction increases..$^{57,58}$

The kinetics of the MB photodegradation of all the samples is shown in Fig. 8. The catalytic rate constants of the MB degradation by bare $\mathrm{ZnS}$, CuS and $\mathrm{CuS} / \mathrm{ZnS}$ nanocomposites are shown in Table 1, which are calculated by the linear fitting of $\ln \left(C_{0} / C_{t}\right)$ versus irradiation time. ${ }^{59}$ The apparent rate constant $\left(K_{\text {app }}\right)$ value was determined for the photocatalyst. In the case of bare $\mathrm{ZnS}$ and CuS, the value of $K_{\text {app }}$ was 0.0046 and 0.1300 $\mathrm{min}^{-1}$, respectively. Whereas, when the composite of $\mathrm{ZnS} / \mathrm{CuS}$ was used as the photocatalyst, the value of $K_{\text {app }}$ increased to $0.4598,0.5848,0.3333$ and $0.2513 \mathrm{~min}^{-1}$ for ZC1, ZC2, ZC3 and ZC4, respectively. The $K_{\text {app }}$ value increased from 0.4598 to $0.5848 \mathrm{~min}^{-1}$ with the addition of CuS concentrations from 
Table 2 Comparison of photocatalytic performance of recently reported ZnS composites and this work

\begin{tabular}{|c|c|c|c|c|c|c|c|}
\hline S. no. & Material & Morphology & Illumination source & Target dye & Dye deg. (\%) & Time (min) & Ref. no. \\
\hline 1 & $\mathrm{ZnS}-\mathrm{GO}$ & Nanosheets & Sunlight & Methylene blue & 86 & 150 & 67 \\
\hline 2 & GQD/ZnS & Nanobelts & Xenon lamp & Rhodamine B & - & 40 & 68 \\
\hline 3 & $\mathrm{ZnS} / \mathrm{CdS} / \mathrm{Ag}_{2} \mathrm{~S}$ & Nanoparticles & Sunlight & Congo dye & 98 & 120 & 69 \\
\hline 4 & $\mathrm{CdS} / \mathrm{ZnS}$ & Quantum dots & Xenon lamp & Methylene blue & 88 & 180 & 70 \\
\hline 5 & $\mathrm{ZnO} / \mathrm{ZnS}_{x} \mathrm{Se}_{1-x}$ & Nanowire & Xenon lamp & Methylene blue & - & 180 & 71 \\
\hline 6 & $\mathrm{ZnO} / \mathrm{ZnS}$ & Hollow structures & UV lamp & $p$-Chlorophenol & 90 & 120 & 72 \\
\hline 7 & ZnS/GAs & 3D nanosheets & Xenon lamp & Methylene blue & 98 & 60 & 73 \\
\hline 8 & $\mathrm{ZnO} / \mathrm{ZnS}$ & Nanowire & $\mathrm{Hg}$-arc lamp & Methylene orange & 92 & 40 & 74 \\
\hline 9 & $\mathrm{Zn}_{x} \mathrm{Cd}_{1-x} \mathrm{~S} / \mathrm{rGO}$ & Layered structure & Xenon lamp & Methylene blue & 98 & 120 & 75 \\
\hline 10 & $\mathrm{CuS} / \mathrm{ZnS}$ & Nanorods & $\begin{array}{l}\text { Xenon lamp, MAX-303, } \\
\text { Asahi spectra }\end{array}$ & Methylene blue & 95 & 05 & This work \\
\hline
\end{tabular}

$0.025 \mathrm{M}$ to $0.050 \mathrm{M}$. When the CuS concentration was increased from $0.075 \mathrm{M}$ to $0.1 \mathrm{M}$, the $K_{\text {app }}$ value decreased from 0.5848 to $0.2513 \mathrm{~min}^{-1}$. The kinetic data obtained by the pseudo-firstorder model, such as $K_{\text {app }}$, the corresponding correlation coefficients $\left(R^{2}\right)$ and the maximum dye degradation in the presence of $\mathrm{CuS} / \mathrm{ZnS}$ composites are presented in Table 1. Comparison of photocatalytic performance was made between the materials developed in this work and other reported $\mathrm{ZnS}$ composites, as shown in Table 2. The CuS/ZnS composites showed 95.51\% degradation in $5 \mathrm{~min}$, which was higher than any other reported materials, indicating the enhanced photocatalytic activity under visible light illumination.

Based on the above results, the photocatalytic mechanism for the $\mathrm{CuS} / \mathrm{ZnS}$ heterojunction can be explained as follows:. ${ }^{\mathbf{6 0}, \mathbf{6 1}}$ Upon visible light irradiation, CuS is excited and the electronhole pairs are produced. According to the interfacial charge transfer (IFCT) mechanism, ${ }^{62}$ the electrons in CuS migrate to the conduction band (CB) of $\mathrm{ZnS}^{63,64}$ This transfer process is thermodynamically favourable because the $\mathrm{CB}$ of $\mathrm{CuS}$ is higher than that of ZnS after equilibrium, as shown in Fig. 9. Meanwhile, the photogenerated holes that remain in the valence band of CuS are positioned at much higher values than those of the VB of ZnS. Therefore, the holes produced within the material cannot migrate to $\mathrm{ZnS}$, and therefore did not oxidize $\mathrm{H}_{2} \mathrm{O}$. ${ }^{65,66}$

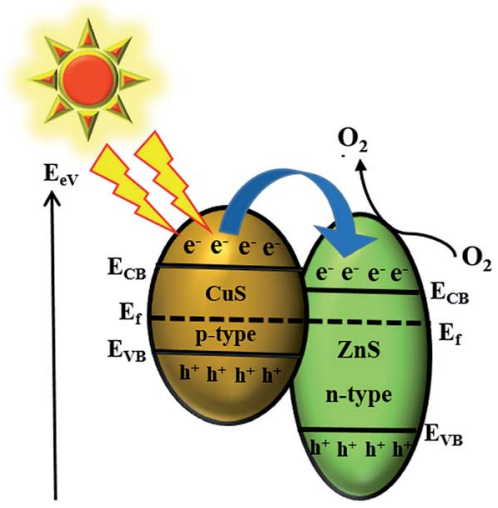

Fig. 9 Schematic mechanism of electron-hole pair separation in the p-type CuS/n-type ZnS heterojunction.

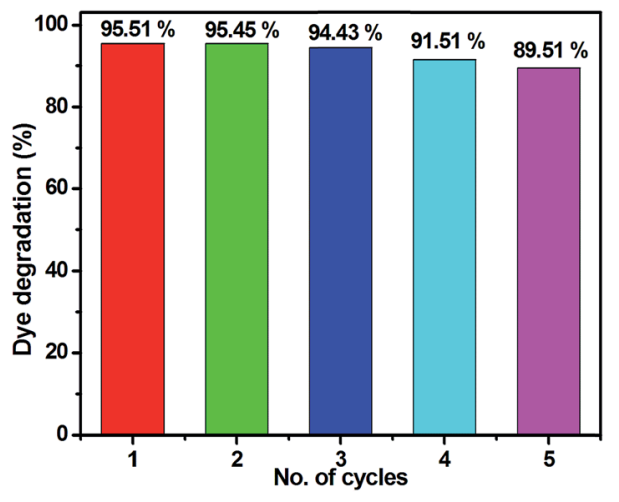

Fig. 10 Reusability of the photocatalyst.

The reusability of the ZC2 photocatalyst was analysed to detect the stability of the material. After the photocatalysis experiment, the catalyst was separated from the reaction mixture by centrifugation. As shown in Fig. 10, the catalyst did not exhibit any significant loss after five cycles of the degradation of MB under the same conditions. The obtained degradation values were $95.51,95.45,94.43,91.51$ and $89.51 \%$ for the first, second, third, fourth and fifth cycles, respectively. The results confirm that the obtained $\mathrm{CuS} / \mathrm{ZnS}$ photocatalyst shows good stability and sustainability. In addition to that, we have investigated the structural property of the used catalyst (ZC2) by XRD and XPS analysis, as shown in Fig. S1 (ESI $\dagger$ ). It can be clearly observed that the phase and structure of the CuS/ZnS composite remained unchanged after the photocatalytic cycles, suggesting that the sample is stable in the present photocatalytic degradation process. The photocatalytic structural stabilities were also investigated by XPS spectra. From the spectra, it is clear that the binding energies of $\mathrm{Zn} 2 \mathrm{p}, \mathrm{Cu} 2 \mathrm{p}$ and $\mathrm{S} 2 \mathrm{~s}$ of CuS/ZnS showed no peak-shift compared to those of the fresh sample.

\section{Conclusion}

$\mathrm{ZnS}$ nanoparticles were decorated on the surface of CuS nanorods by a hydrothermal method. This methodology led to the formation of heterojunctions between p-type CuS and n-type 
ZnS. The investigation of photocatalytic activity indicated that the CuS/ZnS possessed a higher photocatalytic efficiency which was 3 times faster than that of pure $\mathrm{ZnS}$ and CuS. The photocatalytic activity depended on the content of $\mathrm{CuS}$ in the CuS/ZnS composites. The 0.050 M CuS showed the highest photocatalytic activity for the MB degradation. Photogenerated superoxide $\left({ }^{\circ} \mathrm{O}_{2}{ }^{-}\right)$radicals over $\mathrm{CuS} / \mathrm{ZnS}$ were the main reactive species for the degradation of MB. The obtained material is useful for environmental remediation in degrading organic pollutants.

\section{Acknowledgements}

This work was financially supported by (1) Grant-in-Aid for Scientific Research (Chosenteki Houga) (16K14229) from the Ministry of Education, Culture, Sports, Science and Technology (MEXT) of Japan and (2) the cooperative research projects of the Research Institute of Electronics, Shizuoka University. We also thank Prof. K. Murakami, Mr T. Koyama and Mr W. Tomoda, Center for Nanodevices fabrication and analysis for instrumental facilities.

\section{References}

1 E. Pramauro, M. Vincenti, V. Augugliaro and L. Palmisano, Environ. Sci. Technol., 1993, 27, 1790-1795.

2 Y. Zhang, Z. R. Tang, X. Fu and Y. J. Xu, ACS Nano, 2010, 4, 7303-7314.

3 B. Oregan and M. Gratzel, Nature, 1991, 353, 737-740.

4 Z. Wang, H. Wang, B. Liu, W. Qiu, J. Zhang, S. Ran, H. Huang, J. Xu, H. Han, D. Chen and G. Shen, ACS Nano, 2011, 5, 8412-8419.

5 S. Khan, M. Al-Shahry and W. B. Ingler, Science, 2002, 297, 2243-2245.

6 P. Hartmann, D. K. Lee, B. M. Smarsly and J. Janek, ACS Nano, 2010, 4, 3147-3154.

7 Z. Lixin, N. Changhui, J. Hongfang, X. Chunmei, Y. Jibing and Q. Guisheng, Ceram. Int., 2017, 43, 5450-5456.

8 R. Tong, C. Liu, Z. Xu, Q. Kuang, Z. Xie and L. Zheng, ACS Appl. Mater. Interfaces, 2016, 8, 21326-21333.

9 A. Kudo and Y. Miseki, Chem. Soc. Rev., 2009, 38, 253-278.

10 R. Abe, M. Higashi, K. Sayama, Y. Abe and H. Sugihara, J. Phys. Chem. B, 2006, 110, 2219-2226.

11 Q. Li, B. Guo, J. Yu, J. Ran, B. Zhang, H. Yan and J. R. Gong, J. Am. Chem. Soc., 2011, 133, 10878-10884.

12 J. S. Hu, L. L. Ren and Y. G. Guo, Angew. Chem., Int. Ed., 2005, 44, 1269-1273.

13 Z. Fang, S. Weng, X. Ye, W. Feng, Z. Zheng, M. Lu, S. Lin, X. Fu and P. Liu, ACS Appl. Mater. Interfaces, 2015, 7, 13915-13924.

14 Y. Hong, J. Zhang, F. Huang, J. Zhang, X. Wang, Z. Wu, Z. Lin and J. Yu, J. Mater. Chem. A, 2015, 3, 13913-13919.

15 C. Fengjuan, C. Yali and J. Dianzeng, Ceram. Int., 2015, 41, 6645-6652.

16 K. S. Joya, Y. F. Joya, K. Ocakoglu and R. van de Krol, Angew. Chem., Int. Ed., 2013, 52, 10426-10437.

17 Z. Li, W. Luo, M. Zhang, J. Feng and Z. Zou, Energy Environ. Sci., 2013, 6, 347-370.
18 S. Chen, W. Tang, Y. Hu and X. Fu, CrystEngComm, 2013, 15, 7943-7950.

19 Y. Chen, J. C. Crittenden, S. Hackney, L. Sutter and D. W. Hand, Environ. Sci. Technol., 2005, 39, 1201-1208.

20 M. Long, W. Cai, J. Cai, B. Zhou, X. Chai and Y. Wu, J. Phys. Chem. B, 2006, 110, 20211-20216.

21 Z. Liu, Z. G. Zhao and M. Miyauchi, J. Phys. Chem. C, 2009, 113, 17132-17137.

22 M. Lee and K. Young, Nanotechnology, 2012, 23, 194014.

23 Z. Zhang, C. Shao, X. Li, C. Wang, M. Zhang and Y. Liu, ACS Appl. Mater. Interfaces, 2010, 44, 2915-2923.

24 N. Soltani, E. Saiona, W. M. M. Yunus, M. Erfani, M. Navasery, G. Bahmanrokh and K. Rezaee, Appl. Surf. Sci., 2014, 290, 440-447.

25 B. Babu, K. Mallikarjuna, C. V. Reddy and J. Park, Mater. Lett., 2016, 176, 265-269.

26 K. Sunita, K. Sandeep and K. G. Ashok, ACS Sustainable Chem. Eng., 2016, 4, 1487-1499.

27 M. Basu, N. Garg and A. K. Ganguli, J. Mater. Chem. A, 2014, 2, 7517-7525.

28 T. Sakthivel, K. Karthikeyan, K. Sang-Jae and V. Gunasekaran, J. Alloys Compd., 2016, 683, 456-462.

29 M. Basu, A. K. Sinha, M. Pradhan, S. Sarkar, Y. Negishi, Govind and T. Pal, Environ. Sci. Technol., 2010, 44, 63136318.

30 X. Liu, M. T. Mayer and D. Wang, Appl. Phys. Lett., 2010, 96, 223103.

31 X. Meng, G. Tian, Y. Chen, R. Zhai, J. Zhou, Y. Shi, X. Cao, W. Zhou and H. Fu, CrystEngComm, 2013, 15, 5144-5149.

32 Q. Wang, G. Yun, Y. Bai, N. An, Y. Chen, R. Wang, Z. Lei and W. S. Guan, Int. J. Hydrogen Energy, 2014, 39, 13421-13428.

33 X. H. Guan, L. Yang, X. Guan and G. S. Wang, RSC Adv., 2015, 5, 36185-38191.

34 C. Mondal, A. Singh, R. Sahoo, A. K. Sasmal, Y. Negishi and T. Pal, New J. Chem., 2015, 39, 5628-5635.

35 V. Dzhagana, B. Kempken, M. Valakh, J. Parisi, J. KolnyOlesiak and D. R. T. Zahn, Appl. Surf. Sci., 2017, 395, 24-28.

36 X. Chen, F. Zhang, Q. Wang, X. Han, X. Li, J. Liu, H. Lin and F. Qu, Dalton Trans., 2015, 44, 3034-3042.

37 M. F. de Almeida, C. R. Bellato, L. D. L. Miranda and J. L. Milagres, Ceram. Int., 2017, 43, 1843-1852.

38 S. Harish, M. Navaneethan, J. Archana, A. Silambarasan, S. Ponnusamy, C. Muthamizhchelvan and Y. Hayakawa, Dalton Trans., 2015, 44, 10490-10498.

39 J. Yu, J. Zhang and S. Liu, J. Phys. Chem. C, 2010, 114, 1364213649.

40 W. Xnewu, L. Yanan, W. Mingrui, L. Wenjiang, C. Minfang and Z. Yue, New J. Chem., 2014, 38, 4182-4189.

41 U. T. D. Thuy, N. Q. Liem, C. M. A. Parlett, G. M. Lalev and K. Wilson, Catal. Commun., 2014, 44, 62-67.

42 N. Małgorzata, Z. Wojciech, L. Grzegorz, B. Bogusław and Z. Dariusz, Ceram. Int., 2017, 43, 5693-5701.

43 M. Navaneethan, J. Archana, M. Arivanandhan and Y. Hayakawa, Chem. Eng. J., 2012, 213, 70-77.

44 S. Harish, J. Archana, M. Navaneethan, A. Silambarasan, K. D. Nisha, S. Ponnusamy, C. Muthamizhchelvan, 
H. Ikeda, D. K. Aswal and Y. Hayakawa, RSC Adv., 2016, 6, 89721-89731.

45 S. Ameen, M. S. Akhtar, H. K. Seo, Y. S. Kim and H. S. Shin, Chem. Eng. J., 2012, 87, 351-356.

46 Y. Jiaguo, Z. Jun and L. Shengwei, J. Phys. Chem. C, 2010, 114, 13642-13649.

47 S. Harish, M. Navaneethan, J. Archana, S. Ponnusamy, C. Muthamizhchelvan and Y. Hayakawa, Mater. Lett., 2014, 121, 129-132.

48 S. E. Moosavifarda, J. Shamsi, S. Fani and S. Kadkhodazade, Ceram. Int., 2014, 40, 15973-15979.

49 J. Shan, P. Pukkinen, U. Vainio, J. Maijala, H. Jiang, R. Serimaa, E. Kauppinen and H. Tenhu, J. Mater. Chem., 2008, 18, 3200-3208.

50 Y. B. Shao, L. H. Wang and J. H. Huang, RSC Adv., 2016, 6, 84493-84499.

51 C. Feng, X. Meng, X. Song, X. Feng, Y. Zhao and G. Liu, RSC $A d v .$, 2016, 6, 110266-110273.

52 M. Dong, P. Zhou, C. Jiang, B. Cheng and J. Yu, Chem. Phys. Lett., 2017, 668, 1-6.

53 S. Harish, M. Sabarinathan, A. Periyanayaga Kristy, J. Archana, M. Navaneethan, H. Ikeda and Y. Hayakawa, RSC Adv., 2017, 7, 26446-26457.

54 S. Harish, J. Archana, M. Navaneethan, A. Silambarasan, K. D. Nisha, S. Ponnusamy, C. Muthamizhchelvan, H. Ikeda, D. K. Aswal and Y. Hayakawa, RSC Adv., 2016, 6, 89721-89731.

55 M. Q. Yang, Y. Zhang, N. Zhang, Z. R. Tang and Y. J. Xu, Sci. Rep., 2013, 3, 3314-3320.

56 R. Vinoth, P. Karthik, C. Muthamizhchelvan, B. Neppolian and M. Ashokkumar, Phys. Chem. Chem. Phys., 2016, 18, 5179-5191.

57 L. Li, H. Wang, L. Zou and X. Wang, RSC Adv., 2015, 5, 41506-41512.

58 Y. Jiang, J. Hu and J. Li, RSC Adv., 2016, 6, 39810-39817.
59 M. Sabarinathan, S. Harish, J. Archana, M. Navaneethan, H. Ikeda and Y. Hayakawa, RSC Adv., 2016, 6, 109495109505.

60 J. Zhang, Q. Xu, S. Z. Qiao and J. G. Yu, ChemSusChem, 2013, 6, 2009-2015.

61 Y. Zhang, J. Tian, H. Li, L. Wang, X. Qin, A. M. Asiri, A. O. AlYoubi and X. Sun, Langmuir, 2012, 28, 12893-12900.

62 J. Zhang, J. Yu, Y. Zhang, Q. Li and J. R. Gong, Nano Lett., 2011, 11, 4774-4779.

63 H. G. Kim, P. H. Borse, J. S. Jang, E. D. Jeong, O. S. Jung, Y. J. Suh and J. S. Lee, Chem. Commun., 2009, 2, 5889-5891.

64 Y. Zheng, L. Zheng, Y. Zhan, X. Lin, Q. Zheng and K. Wei, Inorg. Chem., 2007, 46, 6980-6986.

65 K. Sinha, P. K. Manna, M. Pradhan, C. Mondal, S. M. Yusuf and T. Pal, RSC Adv., 2014, 4, 208-211.

66 T. Aarthi and G. Madras, Ind. Eng. Chem. Res., 2007, 46, 7-14.

67 H. R. Azimi, M. Ghoranneviss, S. M. Elahi, M. R. Mahmoudian, F. Jamali-Sheini and R. Yousefi, Front. Mater. Sci., 2016, 10, 385-393.

68 S. Ham, Y. Kim, M. J. Park, B. H. Hong and D. J. Jang, RSC Adv., 2016, 6, 24115-24120.

69 K. Kalpana and V. Selvaraj, RSC Adv., 2016, 6, 4227-4236.

70 M. A. Mumin, G. Moula and P. A. Charpentier, RSC Adv., 2015, 5, 67767-67779.

71 Y. Wang, X. Zhan, F. Wang, Q. Wang, M. Safdar and J. He, J. Mater. Chem. A, 2014, 2, 18413-18419.

72 D. A. Reddy, J. Choi, S. Lee, R. Ma and T. K. Kim, RSC Adv., 2015, 5, 18342-18351.

73 D. A. Reddy, J. Choi, S. Lee, R. Ma and T. K. Kim, RSC Adv., 2015, 5, 18342-18351.

74 X. Gao, J. Wang, J. Yua and H. Xu, CrystEngComm, 2015, 17, 6328-6337.

75 X. Wang, H. Tian, X. Cui, W. Zheng and Y. Liu, Dalton Trans., 2014, 43, 12894-12903. 